\title{
Simulation of Gravity Feed Fuel for Aeroplane
}

\author{
Yaguo Lu, Zhenxia Liu \& Shengqin Huang \\ School of Power and Energy, Northwestern Polytechnical University, Xi'an 710072, China \\ Tao Xu \\ Military Representatives office of NDMMP, Xi'an 710025, China
}

\begin{abstract}
Gravity feed is one fuel supply way for aeroplane and the simulation of it is very important. The traditional method to calculate the gravity feed is to assume that only one tank in fuel system supplies the needed fuel to the engine, and then calculated for the single branch. Actually, all fuel tanks compete for supplying fuel and the key problem for gravity feed calculation is to simulate the multiple-branch and transient process. The present paper gives the mathematical model for fuel flow pipe, pump, check valve and the simulation model for fuel tank at first, and then presented a new calculation model for gravity feed fuel of aeroplane fuel system based on the flow network theory and time difference method. The model takes into consideration all fuel tanks and can solve the multiple-branch and transient process of gravity feed. Finally, the thesis gives a numerical example for a certain type of aircraft, achieved the variations of fuel level and flow mass per second of each fuel tanks, the variations of the fuel pressure at the engine inlet, and predicted the maximum time that the aeroplane could fly safely under gravity feed. The numerical example indicts that the method proposed here is intrinsically superior to the traditional methods and is closer to understanding the real seriousness of the fuel supply situation.
\end{abstract}

Keywords: Gravity feed fuel, Element model, Network theory, Multiple-branch, Transient process

\section{Nomenclature}

$Q$

$P \quad$ pressure from a datum at certain points

$P_{s} \quad$ air pressure in fuel tank

$\gamma \quad$ specific gravity of the fluid

$g \quad$ acceleration of gravity

$\rho \quad$ density of fuel

$Z \quad$ elevation from a datum at certain points

$L \quad$ pipe diameter

$D \quad$ pipe length

$f \quad$ friction factor for pipes

$K \quad$ dimensionless experimental coefficient accounting for head losses

$h \quad$ height fuel level from the bottom of tanks

$A(h)$ tank cross-sectional area at $h$

Re Reynolds number

$\mu \quad$ dynamic viscosity of fluid

$P_{i n, o u t} \quad$ pressure at the inlet and outlet of elements

V velocity of fluid 


$\begin{array}{ll}\Delta & \text { pipe roughness } \\ \xi & \text { resistance characteristic constant of pump } \\ a & \text { characteristic constant of check value } \\ P_{c} & \text { crack pressure of check value }\end{array}$

\section{Introduction}

The function of fuel system of aircraft is to store fuel and supply it to the engine under certain flow rate and pressure continuously (Hampus Gavel, Petter Krus, Johan Andersson.)(Hampus Gavel, Petter Krus, Johan Andersson, et al., 2005). Generally, it has two different ways for fuel transfer, the simplest one is by gravity(Hampus Gavel, Petter Krus, Johan Andersson.)(Hampus Gavel, Petter Krus, Johan Andersson, et al., 2005), and another is by pumps. But when flying, it exist the possibility that all the pumps stop working under some situation, such as electrical and mechanical accident. So the simplest method changes to the vital one and the simulation of gravity feed become significant.

Fuel transfer system is a system, which comprises many fuel tanks and much complex pipelines. The traditional method to calculate the gravity feed is to assume that only one tank in fuel system supplies the needed fuel to the engine, and then calculated for the single branch. Actually, gravity feed fuel is a transient process and all fuel tanks compete for supplying fuel according to their ability of fuel supply. The fuel level in fuel tanks decreases when fuel transferring and the ability of one fuel tank for fuel supply decreases also. With these changes, there might have several fuel tanks supplies fuel simultaneously. Thus the fuel supply network is a complex system in which may have many branches. The key problem to calculate gravity feed is the simulation of multiple-branch and transient process. The present paper established a new calculation method for gravity feed of aeroplane fuel system. This model can solve the multiple-branch and transient process simulation of gravity feed fuel and it is intrinsically superior to the traditional methods.

\section{Gravity feed analysis}

The fuel transfer system of a certain aircraft is composed of fuel tanks, fuel pumps, pipes, check valves and other common flow resistance components. When fuel transfers under gravity, the fuel flows spontaneously through the fuel pump and the check valve. Here, the fuel pump is stop operating, which is considered as a resistance element specially. The function of the check valve is to prevent the fuel backward flow between fuel tanks.

Fuel tank supplies fuel according to its ability of gravity feed. There might have some factors effect the ability, which include the air pressure in tanks, $P_{\mathrm{s}}$, the gravitational potential energy of fuel and the resistance of fuel offer pipeline, $h_{\mathrm{w}}$. The pressure in tanks is related to vent system of aircraft and approximately equal in different tanks. The gravitational potential energy of fuel changes with the fuel level, $h$, and the elevation from the bottom of tank to engine inlet, $Z$. Figure 1 is a schematic diagram of gravity feed fuel.

\section{Theory}

\subsection{Flow network theory}

In order to decide the flow rate and pressure drop through the flow network, first, the theory of mass conservation may be imposed at each junction of the flow network. Therefore, the algebraic summation of all flow entering a junction is zero (Wu Dingyi, 1997)(S. M.Chun, 2003)(Cao Lianhua, Zhuang Damin, Ning Chunli, et al, 2002). For the incompressible flow, at a node, the continuity equation is

$$
\sum_{i=1}^{n} Q_{i}=0
$$

Second, the continuity of energy per unit mass may be applied along the same pipeline. The energy potential between two nodes in certain pipe is equal. For the incompressible flow on the same pipeline, the energy balance equation (Victor L. Streeter, E. Benjamin Wylie, Keith W. Bedford, 1998). is

$$
\frac{P_{a}}{\gamma}+\frac{V_{a}^{2}}{2 g}+Z_{a}=\frac{P_{b}}{\gamma}+\frac{V_{b}^{2}}{2 g}+Z_{b}+h_{w}
$$

Where, $P_{\mathrm{a}}, P_{\mathrm{b}}$, and $V_{\mathrm{a}}, V_{\mathrm{b}}$ and $Z_{\mathrm{a}}, Z_{\mathrm{b}}$ represent pressure, velocity and elevation from a datum at certain points, a and b. $h_{\mathrm{w}}$ is frictional head loss. $\gamma$ is specific gravity of the fluid, $g$ is acceleration of gravity.

\subsection{Element Model}

(1) Pipe flow

For the incompressible flow, the pressure drop through a pipe is induced by the energy Equation (2). 


$$
P_{a}-P_{b}=\rho f(Q, D) \frac{8 Q^{2}}{\pi^{2} D^{4}}\left\{\frac{L}{D}+\frac{K}{f(Q, D)}\right\}+\gamma\left(Z_{b}-Z_{a}\right)
$$

Where, $K$ is the dimensionless experimental coefficient accounting for head losses in bend, elbows etc. For the general elbow, $K=0.5$, and the T-type joint, $K=1.0$ (Victor L. Streeter, E. Benjamin Wylie, Keith W. Bedford, 1998). $\rho$ is the density of fluid. $Q$ is volumetric flow rate. $f$ is a friction factor for pipes of circular cross section and can be decided below.

1) laminar flow, $\operatorname{Re} \leq 2000$

$$
f(Q, D)=\frac{64}{\operatorname{Re}}
$$

2) turbulent flow, $\operatorname{Re}>2000$

$$
\frac{1}{\sqrt{f(Q, D)}}=-2.0 \log \left(\frac{\Delta}{3.7 D}+\frac{2.51}{\operatorname{Re} \sqrt{f(Q, D)}}\right)
$$

Where, $\Delta$ is pipe roughness, Re can be expressed by $4 \rho Q / \pi \mu D \cdot \mu$ is dynamic viscosity of fuel.

(2) Fuel Pump flow

The fuel pump is stop operating when fuel transferring under gravity, and it could be considered as a local resistance element. The relationship between flow rate and pressure difference can be expressed by Equation (6).

$P_{\text {in }}-P_{\text {out }}=\xi Q^{2}$

Where, $\xi$ is constant that decide by resistance curve of fuel pump.

(3) Check valve flow

The function of the check valves is to prevent the fuel backward flow between two fuel tanks. The valve remains completely closed until the positive pressure differential exceeds the crack pressure. The relationship between flow rate and pressure difference can be expressed by Equation (7).

$P_{\text {in }}-P_{\text {out }}=a Q^{2}+P_{c}$

Where, $a$ is a constant that decide by characteristic curve which obtained by experimentation, and $P_{\mathrm{c}}$ is the crack pressure.

(4) Fuel tank model

The illustration of model of fuel tank is show in Figure 2. Where, $Z$ is the tank's parameter of location, $P_{\mathrm{s}}$ is air pressure in fuel tank, and $h$ is the fuel level. Because of $Z$ is constant when the fuel system of aircraft has been design finished, $h$ is the only variable that effects the gravitational potential energy of fuel and further effects the ability of gravity feed of fuel tank. We presented the curve for fuel tank, which show the relationship between cross-section area, $A(h)$ and fuel level, $h$. These curves could be used to simulate the changes of fuel level in gravity feed process which detailed in the next part. The curves of the five fuel tank for one aeroplane are illustrated in Figure 3 7.

\subsection{Transient Process Simulation}

This study used the time difference method to simulate the transient process. Based on this method, we divided the whole process of gravity feed into many small time sections, $\Delta t_{1}, \Delta t_{2}, \ldots \Delta t_{n}$. In one time interval $\Delta t_{i}$, it assumed that the whole flow process is steady-state, and the fuel flow rate of each fuel tank is considered as a constant.

On the basis of the difference method, the transient process is simulated below:

1) Define the fuel level of each fuel tank, $h_{i}{ }^{t}$, at the beginning time, $t=t_{0}$, of gravity feed.

2) Define the boundary condition.

3) Network calculation

Calculation for the whole fuel transfer system using flow network theory, obtain the flow rate of each fuel tank, $Q_{i}^{t}$, and the pressure distribution in flow system.

4) Using the fuel tank model to calculate the new fuel level of each fuel tank, $h_{i}^{t+\Delta t}$.

$$
\left\{\begin{array}{l}
\Delta h_{i}^{t}=\frac{Q_{i}^{t} \cdot \Delta t}{A\left(h_{i}^{t}\right)} \\
h_{i}^{t+\Delta t}=h_{i}^{t}-\Delta h_{i}^{t}
\end{array}\right.
$$


Define the new fuel level of each fuel tank when $t=t+\Delta t, h_{i}^{t+\Delta t}$.

Repeat step 3) to step 5), the whole transient process of gravity feed could be simulated.

\subsection{Numerical}

Based on the established method, we calculated the gravity feed for one certain type of aircraft. The aircraft has two (right and left) fuel transfer system, the right system showed in figure 8 transferring fuel for the right engine and the left providing fuel for the left engine, the two system also can supply fuel to one engine simultaneity in addition .

As show in figure 8, the right fuel transfer system has five fuel tanks, and the tank 4 and tank 5 assembled in the wing, the check values which installed in the fuel transfer pipes to prevent the fuel backward flow between fuel tanks.

The right system gravity feed has been simulated in this section with the flight condition: flight height is $7 \mathrm{~km}$ and the engine is 0.5 rated condition. The calculation boundary condition for network is the air pressure in each fuel tanks (approximately equal the atmospheric pressure at $7 \mathrm{~km}$ ) and the fuel flow rate to the engine which is $2800 \mathrm{~kg} / \mathrm{h}$ $\left(0.000997 \mathrm{~m}^{3} / \mathrm{s}\right)$.

We also should give the fuel level in each fuel tanks when calculation. All the fuel tank is assumed filled at the beginning of the calculation and the fuel level for the five fuel tanks could be obtained from the tank relation curves showed in Figure 3 7.

In the simulation, we obtained the variation of fuel level in each fuel tank, the fuel flow rate for each fuel tank and the variation of pressure at the engine inlet. The calculate results are showed in figure $9 \sim 11$.

\section{Analysis}

Figure 9 and Figure 10 give respectively the variations of fuel level and flow rate per second of each five fuel tanks within a period of 16000 seconds. Figure 11 shows the changes of fuel pressure in engine inlet.

As show in Figure 9 and Figure 10, the tank 1, tank 3 and tank 4 all supplies fuel to the engine at different flow rate at the beginning of gravity feed. The flow rates of the three tanks are changes with time, and the flow rate of tank 4 exceeds the flow rate of tank 1 at approximate 9000s. Specially, the tank 2 and tank 5 also begin to offer fuel one after the other with their flow rate increasing and fuel level descending. This all characteristics indict that all fuel tanks compete for supplying fuel according to its' ability of gravity feed respectively.

As show in Figure 11, the fuel pressure in engine inlet decreases gradually. Once the fuel pressure less than the minimum pressure limit in engine inlet, the gravity feed fuel would not ensure the aircraft fly safely under one flight condition. From Figure 11 we could also approximately predict the maximum flight time under gravity feed.

\section{Conclusion}

The thesis established a new calculation model for gravity feed of aeropalne fuel transfer system based on flow network theory and time difference method. This model can solve the multiple-branch and transient process of gravity feed. The model of non-working pump, check valve and fuel tank are also presented.

The results of the simulation of fuel transfer system for one certain type of aircraft show that all fuel tanks compete for fuel supply according to their abilities of gravity feed respectively, but not only one tank offers fuel alone.

\section{Acknowledgements}

The authors would like to acknowledge Prof. Lian Xiaochun and Mr. Xiao Hong for their suggestion and critical review throughout the period of the research.

\section{References}

Cao, Lianhua, Zhuang, Damin, Ning, Chunli, et al.: (2002). Numerical Calculation of Fluid Network System of Fighter. Aircraft Design. 2002,No.4, pp 37-41.

Hampus Gavel, Petter Krus, Johan Andersson, et al.: (2005). Probabilistic Analysis in the Conceptual Phase of an Aircraft Fuel System, 46th AIAA/ASME/ASCE/AHS/ASC Structures, Structural Dynamics \& Materials Confer18-21 April 2005, Austin, Texas.

Hampus Gavel, Petter Krus, Johan Andersson.: Quantification of The Elements in The Relationship Matrix - A Conceptual Study of Aircraft Fuel System. in Proceedings of 42nd AIAA Aerospace Sciences Meeting and Exhibit, Reno, Nevada.

S.M.Chun. (2003). Network Analysis of An Engine Lubrication System. Tribology International. 2003, Vol.36, No.8, pp.609-617.

Victor L. Streeter, E. Benjamin Wylie, Keith W. Bedford. (1998). Fluid Mechanic. McGraw-Hill Companies, Inc. 1998. 
Wu Dingyi. (1997). Network Algorithm of Internal Flow System. Journal of Aerospace Power. 1997, Vol.17, No.6, pp.653-657.

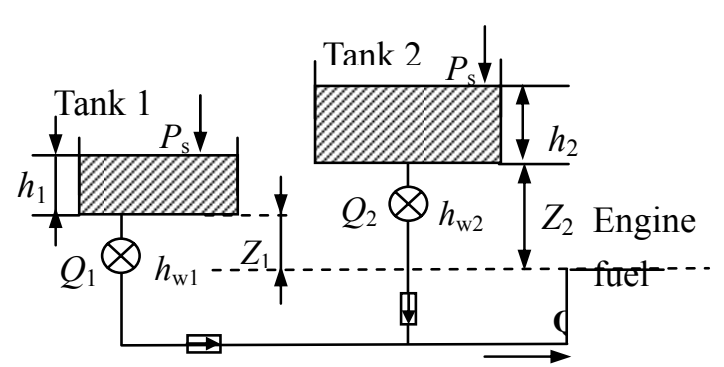

Figure 1. The schematic diagram of gravity feed fuel

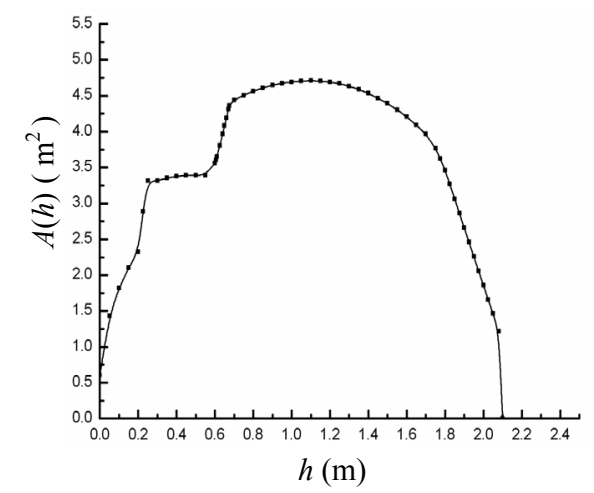

Figure 3. The relation curve for fuel tank 1

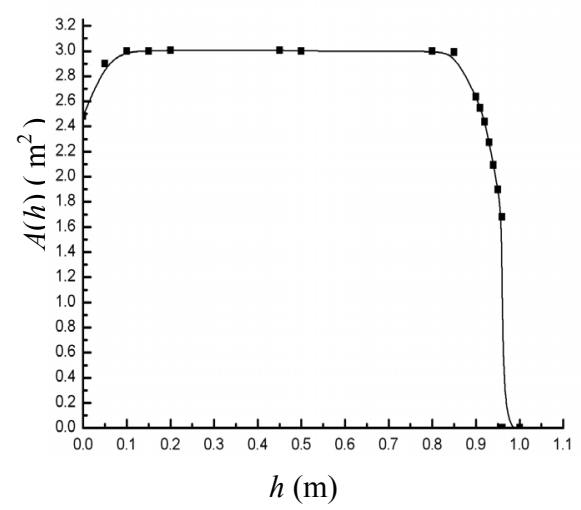

Figure 5. The relation curve for fuel tank 3

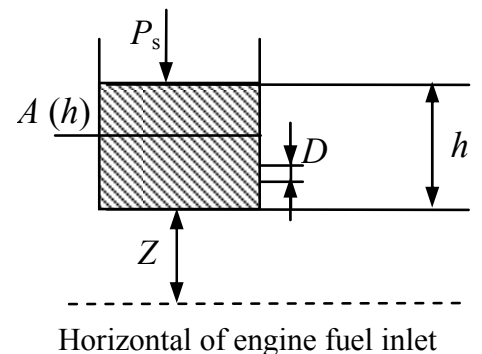

Figure 2. Model of fuel tank

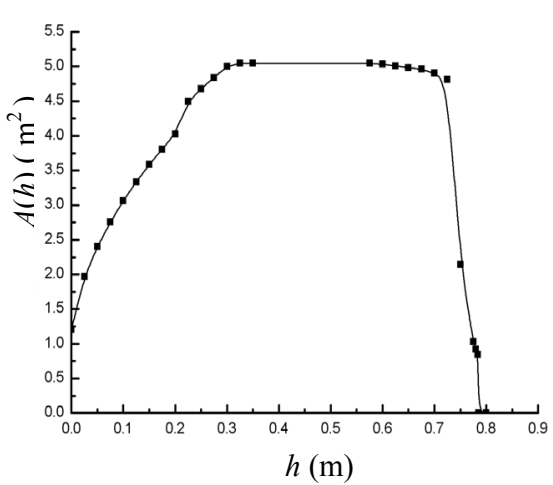

Figure 4. The relation curve for fuel tank 2

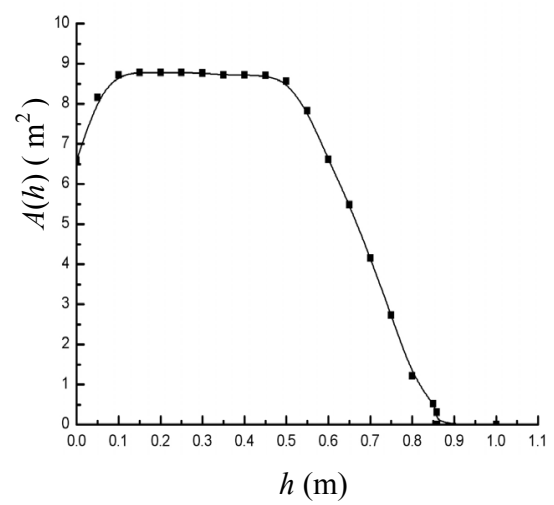

Figure 6. The relation curve for fuel tank 4 


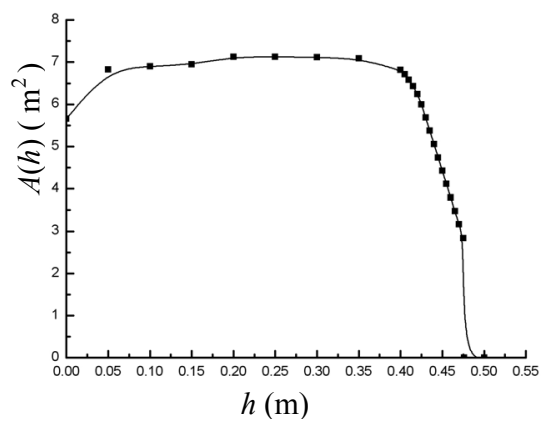

Figure 7. The relation curve for fuel tank 5

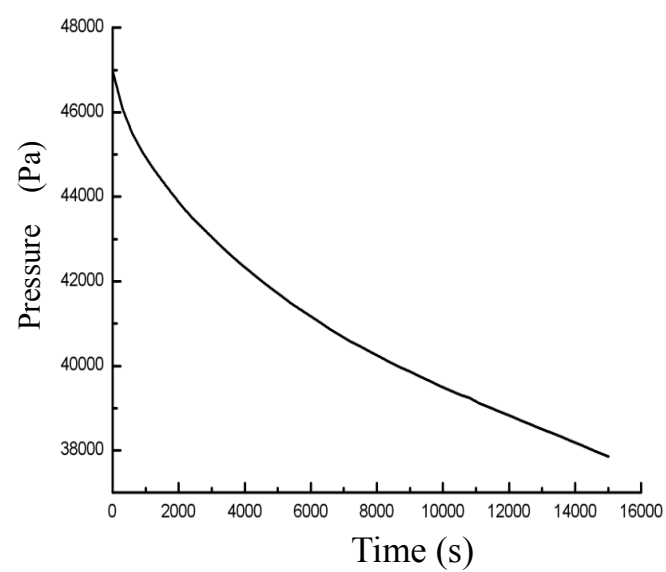

Figure 11. Changes of the fuel pressure at engine inlet

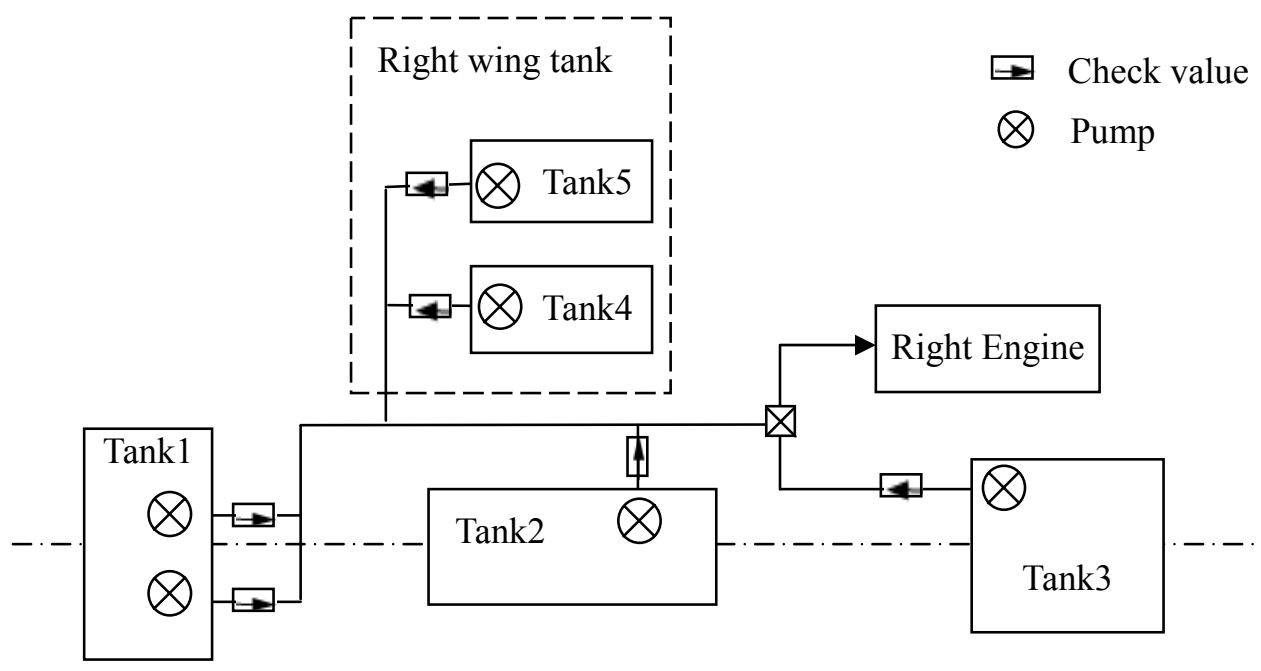

Figure 8. Right fuel transfer svstem for one aircraft

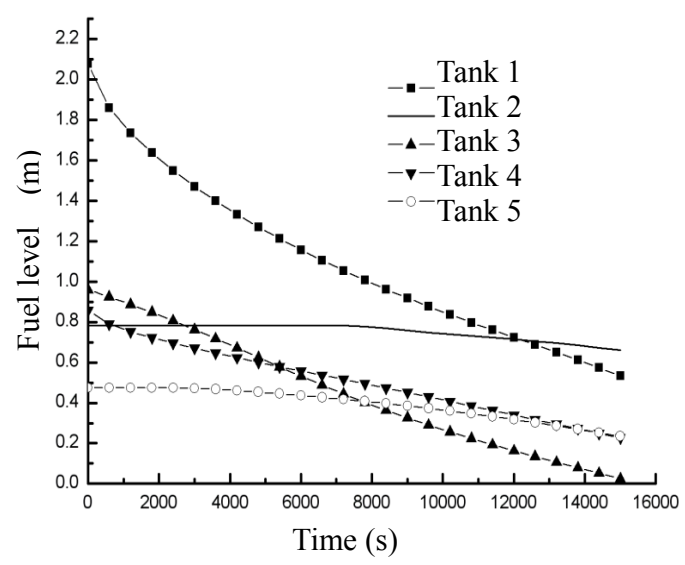

Figure 9. Variation of fuel level in each fuel tank

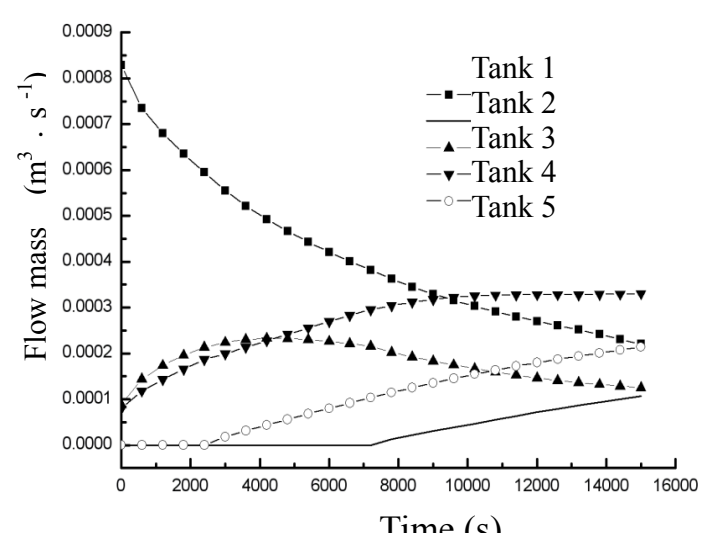

Figure 10. Variation of flow mass of each fuel tank 